\title{
Estratégias heurísticas como meios de ação em atividades de Modelagem Matemática
}

\author{
Lourdes Maria Werle de Almeida
}

\section{Resumo}

No presente artigo dirigimos nossa atenção para os fazeres dos alunos quando desenvolvem atividades de modelagem. Nossa análise tem a intenção de buscar elementos relativos a uma temática reconhecida no campo da resolução de problemas, contudo pouco explorada no âmbito da modelagem matemática: as estratégias heurísticas dos alunos. Nossas argumentações vêm pautadas em um quadro teórico relativo à modelagem matemática e às estratégias heurísticas e em uma pesquisa empírica em que alunos de um curso de Licenciatura em Matemática desenvolvem uma atividade de modelagem matemática. O processo analítico relativo à atividade desenvolvida pelos grupos de alunos nos leva a considerar que as estratégias heurísticas reconhecidas no processo de resolução de problemas também se evidenciam no desenvolvimento da atividade de modelagem matemática. Algumas estratégias parecem intuitivas ou associadas às experiências anteriores dos alunos com modelagem matemática. Outras, entretanto, parecem ser mais específicas e refletem a característica de descoberta, criação associada ao termo heurística.

Palavras-chave: Educação Matemática; Modelagem Matemática; Heurísticas. 


\title{
Heuristic strategies as means of action in Mathematical Modelling Activities
}

\author{
Lourdes Maria Werle de Almeida
}

\section{Abstract}

In this paper we focus our attention on the students' actions when developing modeling activities. Our analysis intends to search for elements related to a recognized theme in the field of problem solving, but little explored in the field of mathematical modeling: the students' heuristic strategies. Our arguments are based on a theoretical framework concerning mathematical modeling and heuristic strategies and on an empirical research in which students of a Mathematics Degree course develop a mathematical modeling activity. The analytical process related to the activity developed by the students leads us to consider that the heuristic strategies recognized in the problem solving process are also evident in the development of the mathematical modeling activity. Some strategies seem intuitive or associated with students' previous experiences with mathematical modeling. Others, however, appear to be more specific and reflect the characteristic of discovery, creation associated with the term heuristic.

Keywords: Mathematics Education; Mathematical Modelling; Heuristics. 


\section{Introdução}

As discussões relativas às ações dos alunos no desenvolvimento de atividades de modelagem matemática têm merecido atenção dos professores e pesquisadores no âmbito da Educação Matemática. Algumas configurações para atividades dessa natureza, bem como, a esquematização dos chamados ciclos de modelagem matemática vêm sendo reconhecidas e usadas pelos professores nos diferentes níveis de escolaridade.

No presente artigo dirigimos nossa atenção para os fazeres dos alunos quando desenvolvem atividades de modelagem. Nossa análise tem a intenção de buscar elementos relativos a uma temática reconhecida no campo da resolução de problemas, contudo pouco explorada no âmbito da modelagem matemática: as estratégias heurísticas dos alunos.

Considerando a caracterização de heurísticas no campo da Educação Matemática, bem como algumas pesquisas desenvolvidas em âmbito internacional e relativas ao uso de estratégias heurísticas em atividades de modelagem matemática, realizamos uma pesquisa empírica em que atividades dessa natureza foram desenvolvidas por alunos do quarto ano de um curso de Licenciatura em Matemática na disciplina de Modelagem Matemática na Perspectiva da Educação Matemática.

O processo analítico relativo às ações dos alunos nestas atividades visa elucidar algum entendimento com relação aos desdobramentos para o desenvolvimento da atividade de modelagem quando algumas estratégias heurísticas podem ser caracterizadas nestas ações.

\section{Modelagem Matemática}

Embora diferentes perspectivas e concepções relativas à modelagem no campo da Educação Matemática sejam compartilhadas, parece haver um consenso em torno da assertiva de Pollak (2015) que considera que a ideia central sempre é "formular uma situação-problema, decidir o que manter e o que ignorar na criação de um modelo matemático, fazer uso de matemática na situação idealizada a partir de uma situação da realidade, e então decidir se os resultados podem, em alguma medida, ser uteis para entender a situação original” (Pollak, 2015, p. 267).

O que tem gerado diálogos que, às vezes convergentes e outras vezes pautados em perspectivas diversas, refere-se às possibilidades de ação daqueles que desenvolvem as atividades de modelagem matemática. Conforme sugere Almeida (2018), o entendimento compartilhado por Pollak abrange a perspectiva de que atividades de modelagem matemática envolvem o uso da linguagem matemática para quantificar situações-problema ou fenômenos da realidade e analisar seu comportamento e que a modelagem matemática pode indicar bons encaminhamentos para resolver problemas em que a matemática é usada para fomentar o entendimento de situações da realidade. 
Alinhado com essas argumentações, o presente artigo pauta-se em um entendimento compartilhado em Almeida (2010), de que uma atividade de modelagem matemática tem início em uma situação inicial (situação-problema) e pode se dizer concluída em uma situação final (resposta para o problema identificado na situação inicial). O caminho entre estes dois extremos é revestido de ações que, previamente definidas ou que emergem na caminhada, são relevantes para o sucesso no desenvolvimento da atividade.

A ideia de um caminho a que nos referimos no presente artigo traz para reflexão estratégias e possibilidades de ação, especialmente quando a modelagem é incorporada em aulas de Matemática em que os fazeres dos alunos, para além de produzir uma solução para o problema, também são associados às suas aprendizagens ao percorrer esse caminho.

De forma geral, o que esse caminho deve incluir vem sendo incorporado nos chamados ciclos de modelagem matemática. Referimo-nos neste texto a um ciclo apresentado em GAIMME (2016) e que tem a intenção de explicitar prováveis, ou talvez desejáveis, ações dos alunos quando desenvolvem uma atividade de modelagem matemática.

Figura 1: Ciclo de modelagem matemática

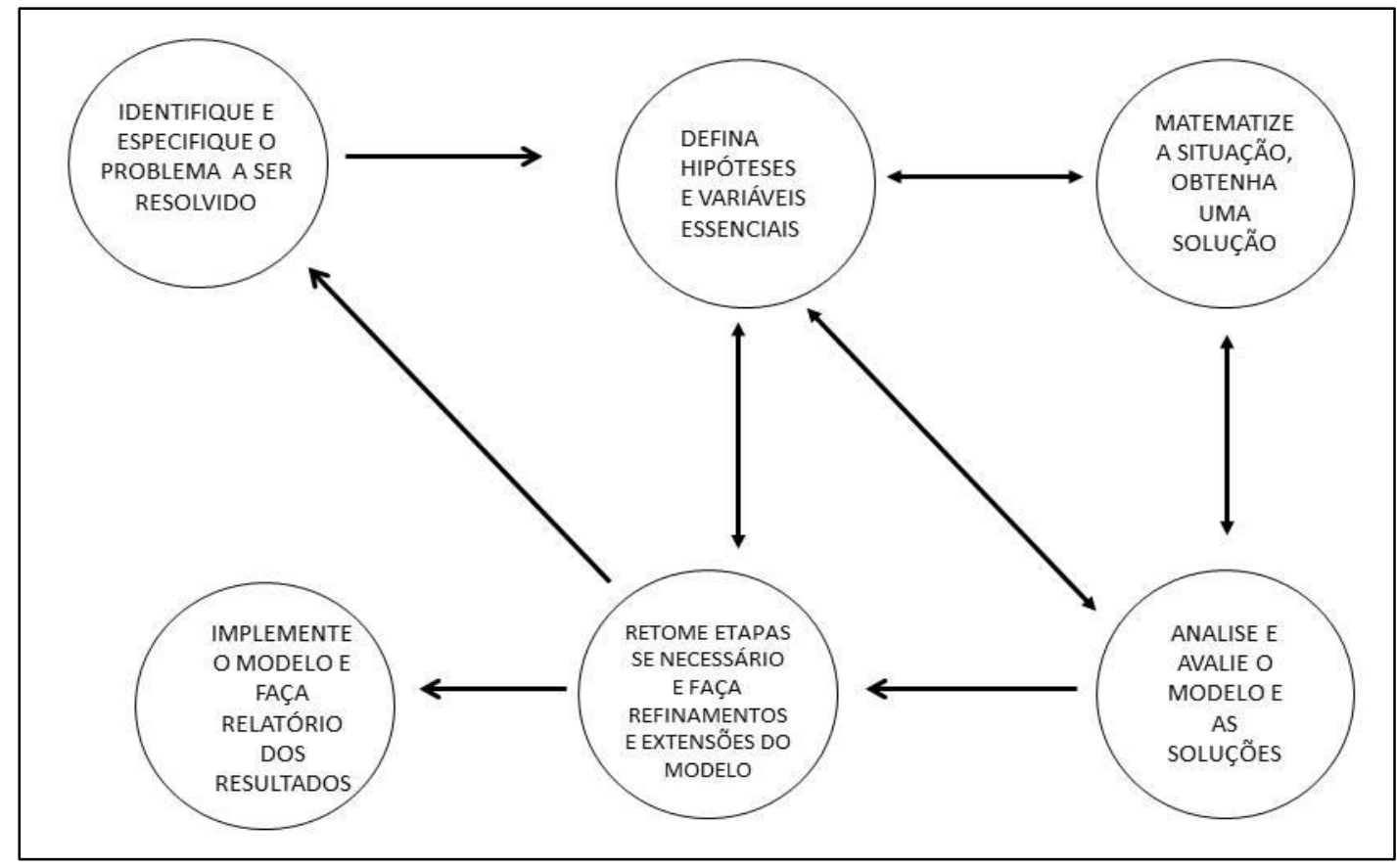

Fonte: adaptado de GAIMME (2016)

Considerando os propósitos do presente artigo, o desafio é identificar os fazeres dos alunos nas diferentes etapas do ciclo associada ao desenvolvimento de uma atividade de modelagem matemática. A orientação para essa identificação busca elementos em assertivas reconhecidas no campo da resolução de problemas, contudo pouco exploradas no âmbito da modelagem matemáticas: as estratégias heurísticas dos alunos.

A busca pela identificação de heurísticas dos alunos ao desenvolver atividades de modelagem matemática já tem apontado para alguns resultados em âmbito internacional, 
como é o caso dos trabalhos de Stender (2018), Stender (2019) e Stender (2018 a). Em sintonia com essas pesquisas, no presente artigo analisamos as ações de dois grupos de alunos ao desenvolverem uma atividade de modelagem matemática.

\section{Estratégias Heurísticas}

O termo heurística tem origem na palavra grega heurisko que é associada a significados como o das expressões Eu encontrei, Eu descobri e inclusive à palavra Eureka apresentada por Arquimedes, sinalizando sempre uma descoberta de alguém em relação a algo (ROTT, 2015). No âmbito da Educação Matemática muito do que se entende por heurística tem sua origem nas ideias do matemático húngaro George Pólya, reconhecido como o precursor da resolução de problemas. Pólya (1945), embora não apresente expressamente uma definição de heurísticas, considera que a "ideia central da heurística é indicar os métodos e as regras relativas à descoberta e à invenção” (p. 112). E, no que se refere à resolução de problemas, Pólya afirma que olhar para as heurísticas nos viabiliza identificar "operações mentais típicas do processo de resolução de um problema” (p. 129).

Kilpatrick (1967, apud Rott, 2015) pondera que a definição de heurísticas certamente envolve dispositivos, técnicas e regras essenciais que incrementam a resolução de um problema. Neste sentido, uma heurística pode ser algo essencialmente provisório, sem garantia de que produza um resultado efetivo na produção de uma resposta para o problema, mas reflete os propósitos daquele que busca a resposta ao traçar e percorrer um caminho de busca.

Esse encaminhamento sugerido por Kilpatrick tem tido reflexos e aprofundamentos, especialmente pelos que se direcionam por uma linha cognitivista para se referir às heurísticas dos alunos, como é o caso de Schoenfeld (1985) que se refere às estratégias heurísticas como regras fundamentais relativas à resolução de um problema, sugestões gerais que auxiliam o aluno a entender um problema e definir procedimentos em busca da solução.

Neste contexto, a própria ideia do que é um problema também merece atenção. Nosso entendimento a esse respeito vem pautado nas argumentações de Dörner (1976). Esse autor afirma que para caracterizar um problema temos que considerar que: um indivíduo se confronta com um problema quando, por alguma razão, se depara com uma situação indesejada (problemática) e não tem meios, a priori definidos, para transformá-la numa situação esperada (que seja confortável). Assim Dörner (1976) defende que um problema é uma situação em que o sujeito identifica qual é seu objetivo, mas não identifica meios para atingir esse objetivo.

Por considerar que os meios para atingir o objetivo, ou seja, resolver o problema são revestidos de estratégias heurísticas, Polya (1945) caracteriza essas estratégias como ferramentas do pensamento matemático para orientar os alunos na busca de uma solução. Hoon et al. (2013), levando em consideração a caracterização de George Pólya, ponderam que 
estratégias heurísticas são relativas a procedimentos experimentais tais como: tentativa e erro, busca de padrões e definição de hipóteses; práticas que, para identificar como seguir em frente, voltam-se a olhar para trás, identificando o que já foi feito e como se pode seguir; práticas que para resolver um problema, consideram útil dividir o problema e resolver subproblemas separadamente. Este delineamento para as estratégias heurísticas tem recebido complementações e interpretações nos últimos tempos (Stender, 2018, 2018ª; 2019; entre outros).

Novotoá et al. (2015) também interpretam as ideias de Pólya e identificam seis grupos de ações relativas a estratégias heurísticas identificadas na resolução de um problema em matemática. A saber, as estratégias: (1) definidas a partir de analogias; (2) simulação do tipo tentativa e erro; (3) uso de experimentações; (4) reformulação do problema, visando identificar partes do problema e, muitas vezes, fragmentações do problema; (5) traçado de figuras ou representações gráficas; e(6) uso de funções e seus gráficos para esboçar a resposta de um problema.

No âmbito da Modelagem Matemática, particularmente, Stender (2018; 2019) tem caracterizado estratégias heurísticas dos alunos em atividades de modelagem. Stender (2018), a partir de pesquisas empíricas, sugere que estratégias heurísticas em atividades de modelagem matemática podem consistir em: organizar o material relativo ao problema; usar o que o autor chama de memória de trabalho, combinando representações, usando simetrias, criando subproblemas; pensar grande, no sentido de não estabelecer limites rígidos para a abrangência dos procedimentos; buscar generalizações; usar o que já sabe de outros problemas ou de outras atividades, em consonância com a ideia de usar analogias; determinar o que o autor chama de aspectos funcionais, e estão associados à definição de encaminhamentos específicos como incrementar os dados usados, ou discretizar a situação; organizar o trabalho, indo e voltando, mantendo a abordagem ou mudando-a se considerar adequado.

No presente texto valemo-nos de uma pesquisa empírica para investigar quais estratégias heurísticas podemos identificar no desenvolvimento de uma atividade de modelagem matemática e que desdobramentos estas estratégias geraram para a obtenção da solução do problema associada a essa atividade.

\section{Apresentação dos dados}

A atividade de modelagem matemática que subsidia nossas argumentações foi desenvolvida por alunos do quarto ano do Curso de Licenciatura em Matemática e ministrada pela autora desse artigo no ano de 2018. Nessa disciplina estavam matriculados 19 alunos que formaram 04 grupos para desenvolver a atividade.

O tema Biometria da Iris Humana foi sugerido aos alunos pela professora e despertou o interesse. A partir de uma aula em que a temática foi discutida com os alunos a partir de 
textos de revistas e de informações disponíveis na internet, os grupos foram reconhecendo que o que fariam nessa atividade de modelagem matemática era criar um modelo para determinar quais pessoas teriam acesso a um determinado espaço (como um banco, um clube, ou um local de trabalho, por exemplo) usando esse modelo e quais não teriam acesso.

Ficou acordado que cada grupo desenvolveria um sistema de reconhecimento pela íris em que os alunos do grupo teriam acesso a um determinado local e os demais não teriam acesso. Assim, foram desenvolvidos 04 sistemas de reconhecimento na turma. No presente artigo, considerando a extensão do texto, são apresentadas as atividades de dois desses grupos. Cada grupo desenvolveu sua atividade durante três dias de aula, sendo que em cada dia a aula era de uma hora e 50 minutos, envolvendo a coleta de dados, a obtenção do modelo e a apresentação da atividade para todos os alunos da turma.

Nesse artigo utilizamos dados que constam nos relatórios dos alunos, anotações em diário de campo realizadas durante as aulas, bem como transcrição de conversas com esses alunos durante o desenvolvimento da atividade e registrada em gravações de áudio ou de vídeo. Inicialmente, é apresentada a descrição da atividade como entregue pelos grupos e, a seguir, as análises a partir das anotações e das gravações (áudio e vídeo) das aulas e respectivas apresentações dos alunos, visando elucidar reflexões relativas às estratégias heurísticas dos alunos nessa atividade.

\section{A atividade do grupo 1}

O grupo 1 era composto por seis alunos, todos homens. O grupo denominou o seu trabalho de Sistema de segurança- reconhecimento ocular. Este grupo, ao apresentar seu trabalho para a turma, se empenhou em apresentar, inicialmente, informações técnicas e financeiras relativas à criação e ao uso de um sistema de reconhecimento baseado nos dados da íris humana, conforme consta no Quadro 1.

\section{Quador 1 - Reconhecimento pela Íris}

- A velocidade de leitura da íris depende da velocidade da máquina de leitura da íris e da leitura do banco de dados.

- Os dados não são armazenados em imagens e sim em códigos, eles ficam bem menores e com tamanho aproximado de 512 bytes.

- O tamanho do disco de armazenamento de dados não é precisa ser muito grande.

- Baseado nisso, para armazenamento de dados de um milhão de pessoas, é necessário um disco com capacidade de $512 \mathrm{~GB}$.

- Na realidade, é preciso um investimento maciço nesse tipo de segurança.

- Comparado com as leituras biométricas, o reconhecimento pela íris é

especialmente mais caro, mas também mais seguro.

- Quando a íris é totalmente mapeada, a sua codificação chega a ter 400 pontos de reconhecimento.

- O sistema de reconhecimento biométrico tem cerca de 60 pontos.

- Cada uma das estações leitoras de íris custa cerca de 8000 dólares.

Fonte: Informações usadas pelos alunos 
Considerando essas informações, bem como as discussões da aula em que a professora apresentou a temática aos alunos, este grupo formalizou sua intenção nessa atividade de modelagem matemática: sem 8 mil dólares e com a finalidade de criar um sistema de segurança baseado no reconhecimento pela íris, iniciamos a nossa modelagem matemática da situação.

O processo de coleta de dados e a manipulação desses dados nesta atividade foram realizados pelos alunos, inicialmente, por fotografias com o celular e, passando tais imagens para o software GeoGebra na etapa de matematização da situação. Esse processo foi descrito pelos alunos conforme indica o Quadro 2.

Quadro 2- Sobre a coleta de dados

- Os dados foram obtidos a partir da análise da área da íris, da área da pupila e da distância entre os centros da íris e da pupila.

- A análise dos olhos dos membros do grupo se deu com a utilização do software GeoGebra como ferramenta para as leituras das imagens.

Descrição da coleta de dados pelos alunos

Para subsidiar a matematização da situação, o grupo definiu duas hipóteses: $\mathrm{H}_{1}$ : $\mathrm{O}$ tamanho da pupila se mantém constante, pois é exposto a uma mesma intensidade de luz; $\mathrm{H}_{2}$ : Em qualquer direção, a foto mantém uma mesma constante de proporcionalidade com o mundo real. Os alunos ponderam também que uma simplificação considerada nessa coleta de dados é que todas as fotos utilizadas para obtenção dos dados da pupila e da íris foram tiradas sob um mesmo ângulo. As imagens com os olhos de todos os participantes do grupo já inseridas no software GeoGebra são apresentadas no Quadro 3.

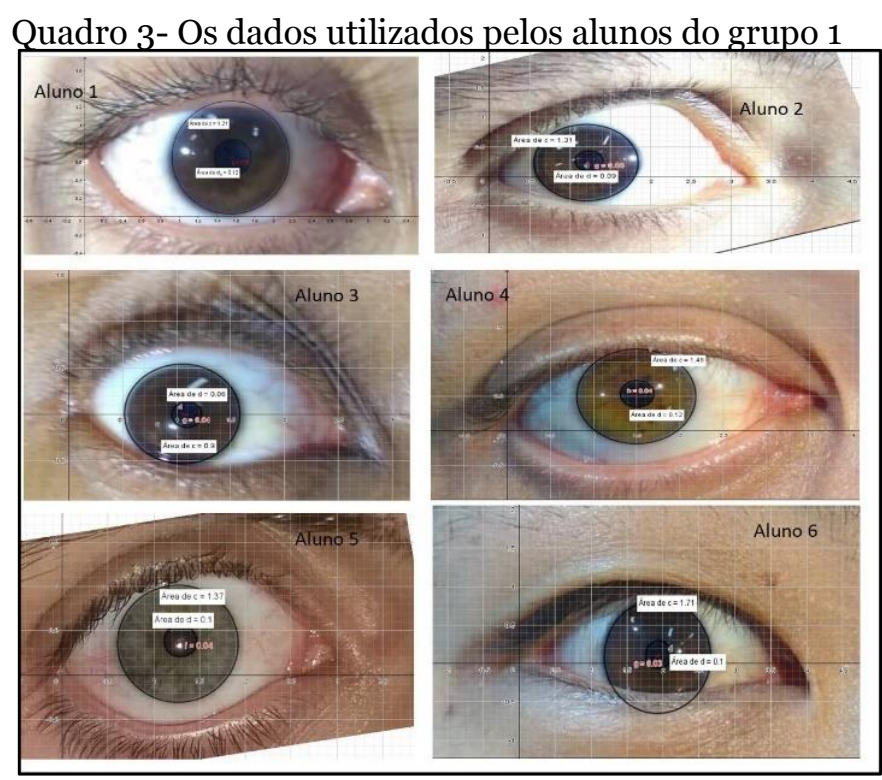

Fonte: Produçãoda Pesquisa 
As variáveis definidas para matematizar a situação foram: $a_{i}$ - área da íris do aluno $i$; $b_{i}$ - área da pupila do aluno $i ; c_{i}$ - distância entre os centros da íris e pupila do aluno $i$. Nos casos em que não estiver especificado o aluno, essas variáveis serão respectivamente $x, y$ e $z$. As medidas dos olhos dos participantes do grupo obtidas pela inclusão e medições no GeoGebra são conforme informa o Quadro 4.

\begin{tabular}{|l|c|c|c|c|c|}
\hline \multicolumn{5}{|c|}{ Quadro 4- As medidas dos olhos dos alunos } \\
\hline & $\begin{array}{c}\text { Tamanho } \\
\text { do olho }\end{array}$ & $\begin{array}{c}\text { Área da } \\
\text { íris (ai) e } \\
(\mathbf{x})\end{array}$ & $\begin{array}{c}\text { Área da } \\
\text { pupila } \\
\text { (bi) e (y) }\end{array}$ & $\begin{array}{c}\text { Distância } \\
\text { entre os } \\
\text { centros } \\
\text { (ci) e (z) }\end{array}$ & $\left(\frac{x}{y}\right)^{2}$ \\
\hline Aluno 1 & 3,1 & 1,21 & 0,12 & 0,02 & 101,6736 \\
\hline Aluno 2 & 3,2 & 1,31 & 0,09 & 0,05 & 211,864 \\
\hline Aluno 3 & 2,8 & 0,9 & 0,06 & 0,04 & 225 \\
\hline Aluno 4 & 3,6 & 1,4 & 0,12 & 0,04 & 152,1111 \\
\hline Aluno 5 & 3 & 1,37 & 0,1 & 0,04 & 187,69 \\
\hline Aluno 6 & 4 & 1,71 & 0,1 & 0,03 & 292,41 \\
\hline
\end{tabular}

Fonte: Informações da Pesquisa

Para desenvolver um modelo matemático - o sistema de segurança baseado em características da íris do olho - este grupo decidiu que seriam definidas $i$ chaves de acesso ao sistema, sendo $i$ o número de usuários com acesso permitido mediante este sistema de segurança. Como o grupo continha seis participantes, decidiram que três seriam considerados usuários e três seriam os chamados intrusos, aqueles que não teriam acesso mediante o uso do sistema de segurança. Definiram que a $i$-ésima chave seria definida como sendo:

$$
f_{i}(x, y, z)=\left|a_{i}-x\right|+\left|b_{i}-y\right|+\left|c_{i}-z\right|+\left|\left(\frac{a_{i}}{b_{i}}\right)^{2}-\left(\frac{x}{y}\right)^{2}\right|
$$

Em que $x, y, z$ são as medidas do olho daquele que está tentando ter acesso ao sistema. Assim, determinaram as chaves de acesso considerando os três alunos do grupo que têm acesso mediante esse sistema e obtiveram os dados conforme mostra o Quadro 5 .

Quadro 5: Chaves de acesso ao sistema

\begin{tabular}{|c|c|c|}
\hline Aluno i & & Chave \\
\hline Aluno 1 & $f_{1}(x, y, z)$ & $|1,21-x|+|0,12-y|+|0,02-z|+\mid\left(101,6736-(x / y)^{2} \mid\right.$ \\
\hline Aluno 2 & $f_{2}(x, y, z)$ & $|1,31-x|+|0,09-y|+|0,05-z|+\left|211,864-(x / y)^{2}\right|$ \\
\hline luno3 & $f_{3}(x, y, z)$ & $|0,9-x|+|0,06-y|+|0,04-z|+\left|225-(x / y)^{2}\right|$ \\
\hline
\end{tabular}

Fonte: Informações da Pesquisa

ISSN 2526-2882 
Para desenvolver a medida que controla o acesso das pessoas ao local mediante esse sistema, ou seja uma medida que identifique os intrusos, o grupo fez a matematização conforme indica o Quadro 6.

Quadro 6: A matematização do controle de acesso

Considere um erro igual a o,5 e os dados do possível intruso:
x corresponde a área da íris;
y corresponde à á rea da pupila;
z a distância entre os centros da pupila e da íris;
O algoritmo para determinar se a pessoa passará ou não pelo sistema de segurança, será
da seguinte forma:
a)Para que a pessoa tenha autorização de entrada concreta, seus dados devem ser tais que
$\mathrm{f}_{1}(\mathrm{x}, \mathrm{y}, \mathrm{z})<0,5$ para todo i= 1, 2 ou 3 ;
b)Caso a) não se verifique, a pessoa terá acesso negado, Ou seja, se $\mathrm{f}_{1}(\mathrm{x}, \mathrm{y}, \mathrm{z})<0,5$
para algum i, a pessoa terá acesso negado.

Fonte: Informações da Pesquisa

Usando esse modelo os alunos verificaram que três dos alunos eram usuários e três eram intrusos mediante o uso do sistema desenvolvido.

Quadro 7: Medida do Controle de acesso

\begin{tabular}{|c|c|c|c|c|c|c|c|c|c|}
\hline & $\begin{array}{c}\text { Tamanho } \\
\text { do olho }\end{array}$ & $\begin{array}{c}\text { Área } \\
\text { da íris } \\
\text { (a) e (x) }\end{array}$ & $\begin{array}{c}\text { Área da } \\
\text { pupila } \\
\text { (b) e (y) }\end{array}$ & $\begin{array}{l}\text { Distância } \\
\text { entre os } \\
\text { centros } \\
\text { (c) }\end{array}$ & $(\mathrm{x} / \mathrm{y})^{\wedge} \mathbf{2}$ & $\begin{array}{c}\text { Chave } \\
\text { para } \\
\text { intruso } \\
1 \\
\end{array}$ & \begin{tabular}{|c|} 
Chave \\
para \\
intrus \\
02 \\
\end{tabular} & \begin{tabular}{|c|} 
Chave \\
para \\
intruso \\
3 \\
\end{tabular} & ERRO \\
\hline $\begin{array}{c}\text { Aluno } 1 \\
\text { (chave 1) } \\
\end{array}$ & 3,1 & 1,21 & 0,12 & 0,02 & 101,6736 & & & & 0 \\
\hline $\begin{array}{c}\begin{array}{c}\text { Aluno } 2 \\
\text { (chave 2) }\end{array} \\
\end{array}$ & 3,2 & 1,31 & 0,09 & 0,05 & 211,864 & & & & 0 \\
\hline $\begin{array}{r}\text { Aluno 3 } \\
\text { (chave 3) } \\
\end{array}$ & 2,8 & 0,9 & 0,06 & 0,04 & 225 & & & & 0 \\
\hline $\begin{array}{c}\text { INTRUS } \\
0 \\
\end{array}$ & NÃO CONS. & $\mathrm{X}$ & $\mathrm{y}$ & $\mathrm{z}$ & $(\mathrm{x} / \mathrm{y})^{\wedge} 2$ & & & & \\
\hline $\begin{array}{c}\text { Aluno } 4 \\
\text { (intruso } \\
1 \text { ) } \\
\end{array}$ & Não CONS. & 1,4 & 0,12 & 0,04 & 152,1111 & 50,6475 & 86,216 & 191,266 & Erro $>0,5$ \\
\hline $\begin{array}{c}\text { Aluno } 5 \\
\text { (intruso } \\
2 \text { ) } \\
\end{array}$ & NÃO CONS. & 1,37 & 0,1 & 0,04 & 187,69 & 59,8829 & 24,254 & 80,976 & Erro $>0,5$ \\
\hline $\begin{array}{c}\text { Aluno } 6 \\
\text { (intruso } \\
\text { 3) }\end{array}$ & NÃO CONS. & 1,71 & 0,1 & 0,03 & 292,41 & 73,4489 & 37,82 & 68,27 & Erro $>0,5$ \\
\hline
\end{tabular}

Fonte: Informações da Pesquisa

Os alunos apresentaram sua conclusão conforme mostra o Quadro 8, a seguir.

Quadro 8: A conclusão dos alunos

Concluímos que o nosso sistema de segurança foi um sucesso, pois, nenhum intruso conseguiu burlar o sistema, ou seja, quebrar a chave.

Fonte: Informações da Pesquisa 


\section{A atividade do grupo 2}

O grupo que desenvolveu essa atividade era composto por quatro alunos, sendo um homem e três mulheres. Os alunos, a partir da aula em que o tema foi proposta pela professora, descreveram o seu problema de interesse como sendo: Criar um algoritmo, (modelo) a partir de dados relativos à íris do olho, que permita determinar se uma pessoa pertence a um determinado grupo ou não.

Os dados relativos à íris consistem em fotos tiradas com o celular dos alunos e inseridas no software Tracker. A hipótese definida pelos alunos foi: o reconhecimento da íris é feito sempre no mesmo lugar, com a mesma incidência de luz, o que implica que a pupila tem sempre a mesma dilatação. Sabendo que, na verdade, a íris humana em geral tem forma elíptica, os alunos fizeram uma simplificação, assumido que a íris é circular. Essa simplificação seria relevante para o processo de matematização da situação. Dentre as diferentes medidas relativas à íris, o grupo optou por considerar na atividade as medidas: a distância entre as extremidades do olho; a área da íris; o raio da pupila. Usando essa hipótese, essa simplificação e as medidas que seriam usadas, os alunos inseriram as imagens do olho dos alunos do grupo na Tracker e determinaram essas medidas usando o próprio software. A imagem dos olhos com as medidas do olho de cada aluno é apresentada no Quadro 9.

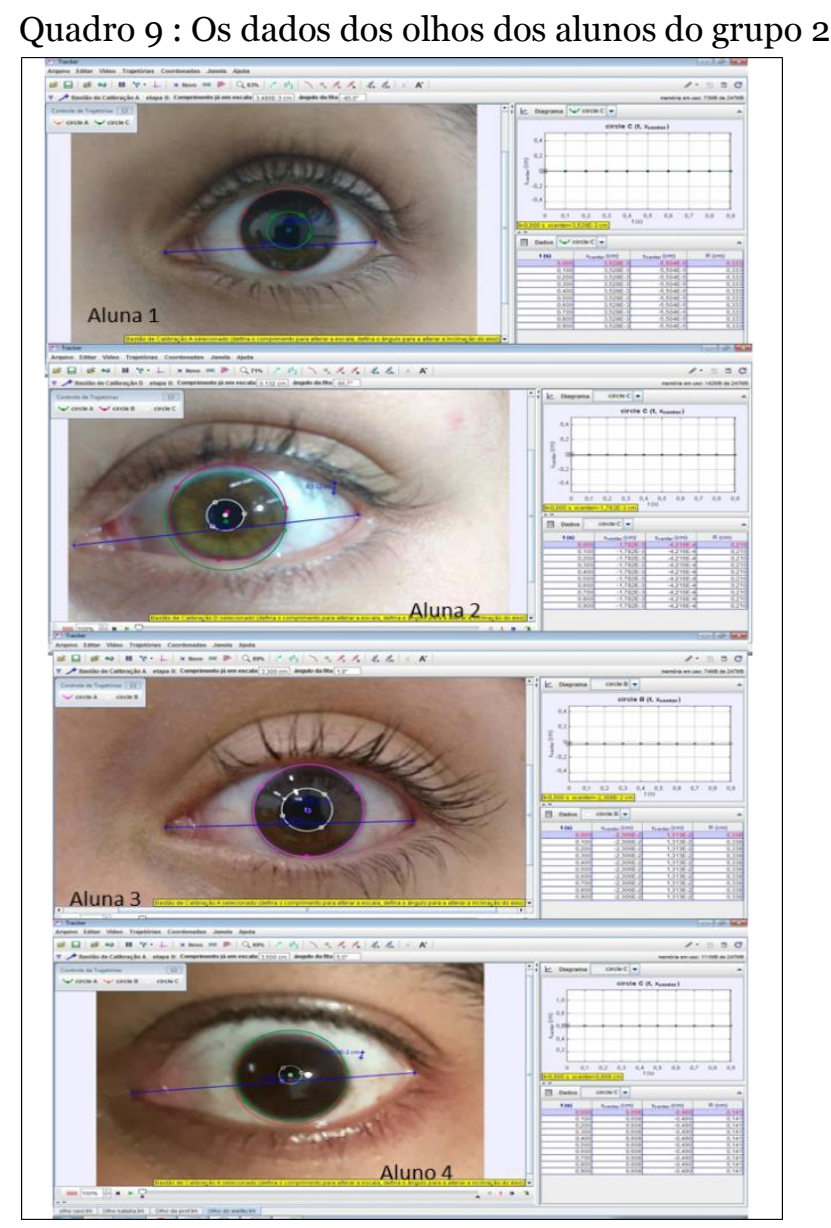

Fonte: Informações da Pesquisa

ISSN 2526-2882

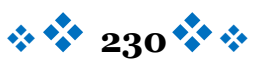


As medidas dos olhos obtidas pelo Tracker constam no Quadro 10.

Quadro 10: as medidas dos olhos dos alunos

\begin{tabular}{|c|c|c|c|c|}
\hline & Aluna 1 & Aluna 2 & Aluna 3 & Aluno 4 \\
\hline Distância entre extremidades & $3,2 \mathrm{~cm}$ & $3,3 \mathrm{~cm}$ & $3,2 \mathrm{~cm}$ & $3,5 \mathrm{~cm}$ \\
\hline Área da íris & $1,79 \mathrm{~cm}$ & $1,68 \mathrm{~cm}$ & $1,21 \mathrm{~cm}$ & $1,39 \mathrm{~cm}$ \\
\hline Raio da pupila & $0,338 \mathrm{~cm}$ & $0,333 \mathrm{~cm}$ & $0,210 \mathrm{~cm}$ & $0,141 \mathrm{~cm}$ \\
\hline $\begin{array}{l}\text { Distância entre centros das } \\
\text { circunferências que contenham a } \\
\text { íris }\end{array}$ & $0 \mathrm{~cm}$ & $0 \mathrm{~cm}$ & $0,132 \mathrm{~cm}$ & 0,08319 \\
\hline Comprimento da íris & $4,74 \mathrm{~cm}$ & $4,6 \mathrm{~cm}$ & $3,89 \mathrm{~cm}$ & $4,17 \mathrm{~cm}$ \\
\hline
\end{tabular}

Fonte: Informações da Pesquisa

Para a matematização da situação os alunos estabeleceram as seguintes variáveis e parâmetros:

Quadro 11: A definição do modelo

$x$ : distância entre as extremidades do olho; $y$ : área da íris; $z$ : raio da pupila

Matriz padrão: $P_{3 \times 3}=\left[\begin{array}{lll}2 & 3 & 1 \\ 2 & 4 & 3 \\ 1 & 2 & 7\end{array}\right] \quad$ Matriz dos dados individuais: $Q_{3 \times 1}=\left[\begin{array}{l}x \\ y \\ z\end{array}\right]$

Fonte: Informações da Pesquisa

As operações usando esses dados foram definidas conforme indica o Quadro 11.

Quadro 11: A definição do modelo

No reconhecimento da íris, nosso algoritmo vai:

a)Captar as medidas $\mathrm{x}, \mathrm{y}$ e $\mathrm{z}$ da pessoa que está tentnado entrar no nosso sistema

b) Fazer uma multiplicação da matriz $P$ pela matriz $Q$

c) Obter a matriz resultante $\mathrm{R}_{3 \times 1}$, em que $\mathrm{a}_{1,1}=\mathrm{A}, \mathrm{a}_{2,1}=\mathrm{B}$ e $\mathrm{a}_{3,1}=\mathrm{C}$

d)Realizar a operação $\frac{A+B}{C}=\boldsymbol{\gamma}$, com os devidos parâmetros obtidoz resnte ultas na

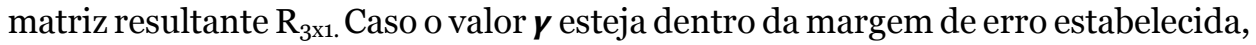
esta pessoa terá acesso ao sistema!

Fonte: Informações da Pesquisa

A medida de erro foi definida pelos alunos conforme mostra o Quadro 12

Quadro 12: A medida de erro

A medida do erro foi desenvolvido a partir do valor, encontrado para a cada um dos indivíduos pertencentes ao grupo. Os valores encontrados foram

$\boldsymbol{Y}_{\text {aluno } 1}=17,14$

$\boldsymbol{Y}_{\text {aluno } 1}=19,01$

$\boldsymbol{V}_{\text {aluno } 1}=21,99$

$\boldsymbol{Y}_{\text {aluno } 1}=19,33$

Duas possibilidades de medida de erro foram estudantos:

Intervalo geral: É considerado pertencente ao grupo aquele em que $\gamma=\in(17,22)$

Intervalos específicos: É considerado pertencente ao grupo aquele em que $\gamma=\epsilon$

$(17,13,17,15)$ ou $\gamma=\in(19,19,02)$ ou $\gamma=\in(19,32,19,34)$ ou $\gamma=\in(21,98,22)$

Fonte: Informações da Pesquisa

ISSN 2526-2882 
Para testar o modelo desenvolvido, o grupo tirou foto do olho da professora e, determinando as medidas e usando o modelo verificaram que ela seria uma invasora, ou seja, não tem acesso ao grupo mediante o sistema de segurança desenvolvido. Estes procedimentos bem como a conclusão dos alunos são apresentados no Quadro 13.

Quadro 13: Testando o modelo desenvolvido e a conclusão

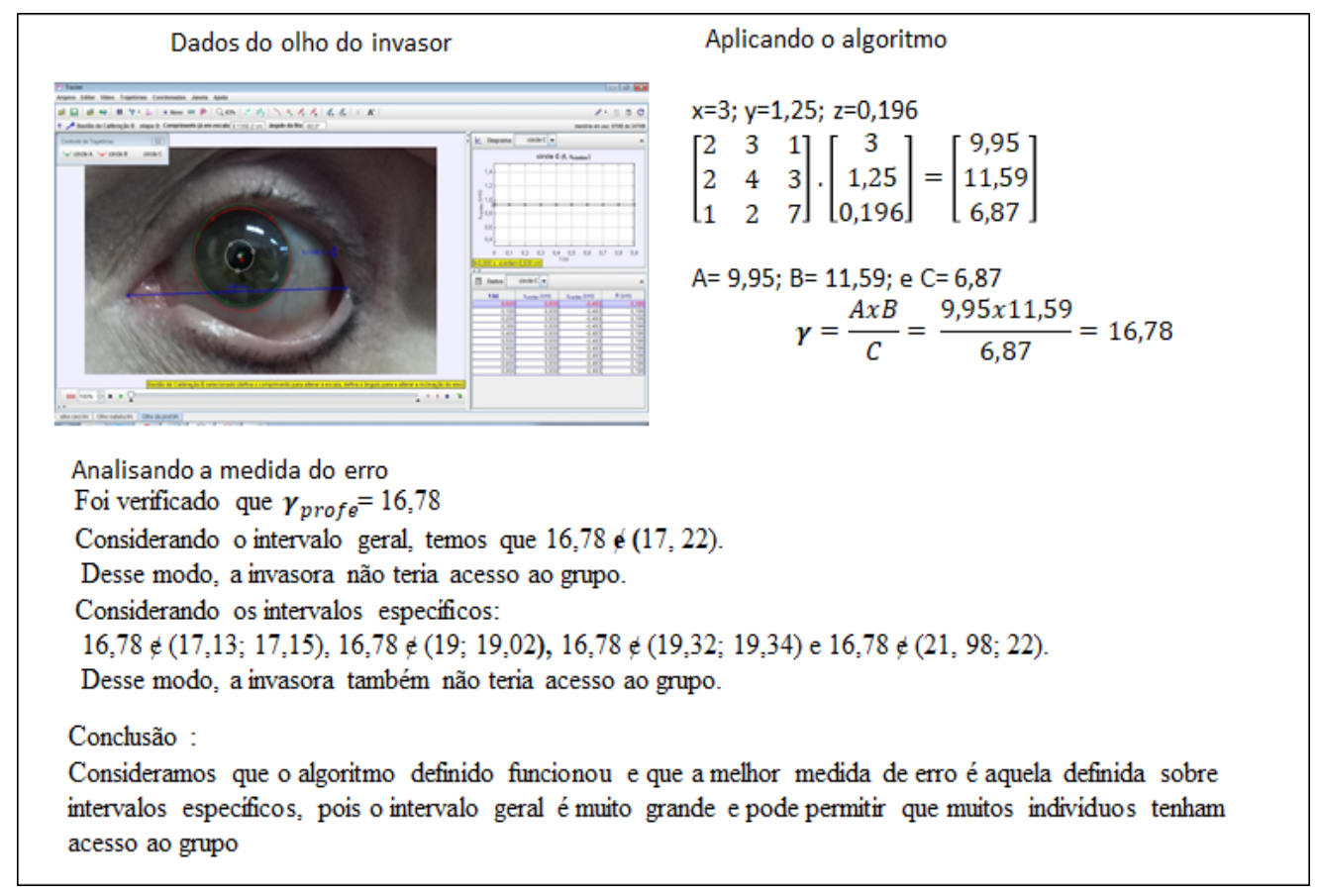

Fonte: Informações da Pesquisa

\section{Discussão e resultados}

As reflexões acerca das estratégias heurísticas dos alunos em atividades de modelagem matemática têm a intenção sinalizar como estas estratégias influenciam o desenvolvimento das atividades realizado pelos alunos.

A temática da atividade em que se pautam as argumentações foi sugerida aos alunos pela professora da disciplina. Os alunos, entretanto, prontamente se interessaram pelo tema e se apropriaram de suas especificidades, sobretudo pelos possíveis encaminhamentos que essa atividade poderia seguir.

As primeiras manifestações dos alunos eram voltadas ao que eles chamavam de falta de conhecimento sobre algoritmos para buscar uma solução para o problema que nesta situação inicial estava se delineando. Todavia, na medida em que o diálogo entre professora e alunos permeava as ações na sala de aula, os diferentes grupos foram tomando suas iniciativas.

A primeira ação no caminho entre a situação inicial (que lhes solicitava a criação de um sistema de reconhecimento pela íris) e a situação final (a apresentação desse sistema testado mediante a identificação de pertencentes e de intrusos a um certo grupo) foi justamente entender o problema. Nos termos que é abordado em Stender (2018), a situação parecia aos 
alunos diversa em relação ao que já tinham feito antes e também deveras complexa. Assim, ainda na etapa de identificar e especificar o problema, a estratégia heurística de pensar grande e não limitar o pensamento a fronteiras foi fundamental para que os procedimentos dos alunos fossem organizados de forma a elucidar como o caminho deveria ser percorrido.

O grupo 1, nesse contexto, investiu, inicialmente, na busca por mais informações sobre a situação, trazendo à baila aspectos como a capacidade do computador que armazena os dados de um sistema de reconhecimento pela íris e os custos de implantação desse sistema, conforme sugere o Quadro 1.

Entretanto, a convicção de que o contexto em que a atividade estava sendo desenvolvida, prontamente lhes trouxe a certeza de que poderiam combinar itens complexos (como é a temática) com o conhecimento que possuíam ou estavam dispostos a acionar enquanto alunos do quarto ano de um curso de Licenciatura em Matemática. Isso sinaliza uma estratégia heurística - uma estratégia do pensamento como sugere Pólya (1945), que se alinha com a definição de propósitos para resolver o problema e o traçado de um caminho a percorrer.

O grupo 2 foi passivo em relação a ideia de buscar informações complementares em relação àquelas disponibilizadas pela professora. Os alunos desse grupo consideraram suficientes as informações que possuíam e seu esforço se direcionou para o processo de coleta de dados relativos à íris e de criação de um modelo matemático. Para a coleta de dados- tirar fotos dos olhos e inserir as imagens no software Tracker ou GeoGebra - os alunos contaram, em um primeiro momento, com a colaboração dos dois alunos de mestrado que participavam das aulas.

O ponto central na atividade é que, a partir dessas imagens, eles escolheriam quais medidas usar e como fazer a matematização da situação usando essas medidas. Neste sentido, as estratégias de definir hipóteses e simplificar a situação foram fundamentais para determinar os encaminhamentos. Todavia, essas estratégias dos alunos confirmam as conjecturas de Kilpatrick (1967, apud Rott, 2015) com relação à provisoriedade de algumas heurísticas. O que se apresenta na descrição da atividade, no presente artigo, é o resultado das discussões e dos ensaios dos alunos para explicitar quais são as hipóteses e qual a simplificação que devem ser explicitadas no desenvolvimento da atividade.

No que se refere à simplificação de considerar a íris como sendo circular, quando na verdade na maior parte das pessoas ela é elíptica, os alunos fizeram ensaios de considerar a íris com forma de elipse, mas como uma das variáveis é a área da íris, decidiram pela forma circular para, na verdade, simplificar os cálculos. No diálogo com a professora apresentaram sua indecisão e suas tentativas de conciliar o cálculo da área da elipse com os demais procedimentos relativos à criação do modelo matemático.

Pode-se conjecturar nesse sentido que a estratégia heurística que guiou os fazeres dos alunos refere-se ofazer o que sabe, fazendo analogias com outros problemas. De fato, os alunos 
de ambos os grupos já possuíam experiências anteriores com modelagem matemática na disciplina e estavam convictos de que as simplificações e as hipóteses determinam a matematização e influenciam a qualidade dos resultados.

O que se pode inferir com relação aos dois grupos é que, realmente, dividiram o problema em subproblemas: primeiro, coletar os dados, ou seja, tirar as fotos dos olhos captando íris e pupila e inserir as imagens no software; segundo, quais medidas escolher, considerando as possibilidades do software escolhido; terceiro, desenvolver o modelo matemático; e, finalmente, testar o modelo.

Neste contexto, os dois grupos organizaram o material e suas estratégias heurísticas funcionaram comoferramentas do pensamento em que os meios usados na busca não estavam a priori definidos. Esses grupos usaram ferramentas matemáticas distintas para construir o modelo. Isso é formidável, pois vem fomentar o que já se discute na literatura de que a matemática, em geral, não pode ser previamente escolhida, mas essa escolha é o coração da modelagem, como afirma Pollak (2015).

O que parece relevante nas estratégias dos alunos é como combinaram as ferramentas matemáticas articulando conceitos, operações e resultados. Neste contexto, o grupo 1 o fez criando o que chamaram de chaves de acesso ao sistema e definindo essas chaves por meio de uma função cujas variáveis são as medidas do olho por eles escolhidas. E o grupo 2 valeu-se de operações com matrizes que, adequadamente combinadas, viabilizaram a criação de um sistema de reconhecimento eficiente e fizeram sua testagem.

O processo analítico da atividade desenvolvida pelos grupos nos leva a considerar que as estratégias heurísticas reconhecidas no processo de resolução de problemas também se evidenciam em diferentes etapas especificadas no ciclo de desenvolvimento da atividade de modelagem matemática. Algumas estratégias parecem intuitivas ou associadas às experiências anteriores dos alunos com modelagem matemática. Outras, entretanto, são específicas para cada atividade e refletem a característica de descoberta, criação associada ao termo heurística, conforme aponta Rott (2015) e são influenciadas pelo problema a ser resolvido na atividade de modelagem matemática.

Neste sentido, as estratégias heurísticas identificadas na pesquisa de Stender (2018) também podem ser identificadas nos fazeres dos grupos de alunos a que nos referimos. O que se pode inferir, sobretudo, é que no que se refere à etapa final indicada no ciclo de modelagem matemática apresentado no presente artigo, é que a implementação do modelo foi efetivada pelos alunos, pelo menos no contexto da sala de aula em que a atividade foi desenvolvida. Esse fato pode ser um indício de que a estratégia heurística de pensar grande faz parte dos fazeres dos alunos e vem ao encontro da caracterização de Pólya referente à caracterização das estratégias heurísticas como ferramentas do pensamento na resolução de um problema. 
Embora se identifiquem esses atos criativos dos alunos, é preciso sublinhar que a intervenção e a orientação da professora foram essenciais para que as estratégias heurísticas dos alunos se convertessem em meios de ação que, efetivamente, colaboraram na produção de modelos robustos e cujos resultados se mostraram adequados para a interpretação e análise do problema em estudo na atividade de modelagem matemática. Na presente pesquisa, essas intervenções não foram objeto de estudo/análise, mas dada sua relevância - que se mostra nas gravações em áudio e vídeo relativas às atividades dos alunos - estudos posteriores deverão ser realizados para investigar esse aspecto.

\section{Referências}

ALMEIDA, L. M. W. Considerations on the use of mathematics in modeling activities. ZDM, 50(1), 19-30, 2018.

ALMEIDA, L. M. W. Um olhar semiótico sobre modelos e modelagem: metáforas como foco de análise. Zetetiké, Campinas, v. 18, número temático, p. 387-414, dez. 2010.

DÖRNER, D. Problemlösen als Informationsverarbeitung. Kohlhammer, Stuttgart. 1976.

GAIMME - Guidelines for assessment \&instructions in mathematics modeling education. NCTM, 2016.

HOON, T. S.; KEE, K. L. ; SINGH, P. Learning Mathematics using heuristic approach. Procedia - Social and Behavioral Sciences 90862 - 869, 2013.

NOVOTNÁ, J. et al. Problem solving in school mathematics based on Heuristic strategies. ERIES Journal vol. 7 no. 1, 2014.

POLLAK, H. O. Introduction: what is mathematical modeling?. In: H. Gould, D. R. Murray, \& A. Sanfratello (Eds.). Mathematical Modeling Handbook (p. 8-11). Bedford: Comap, 2012.

POLLAK, H. O. The Place of Mathematical Modelling in the System of Mathematics Education: Perspective and Prospect. In: G. A. Stillman, W. Blum, \& M. S. Biembengut (Eds). Mathematical Modelling in Education Research and Pratice (p. 265-276). Cham, Switzerland: Springer, 2015.

PÓLYA, G. How to Solve It. Princeton, University Press, 1945.

ROTT, B. Rethinking heuristics - characterizations and vignettes. LUMAT 3(1), 2015.

STENDER, P. Heuristic Strategies as a Toolbox in Complex Modelling Problems. ICME-13 Monographs. Series Editor: Gabriele Kaiser, Faculty of Education, Didactics of Mathematics, Universität Hamburg, Hamburg, Germany, 2019.

STENDER, P. STUHLMANN, S. Fostering Heuristic Strategies in Mathematics Teacher Education. INDRUM 2018, I NDRUM Network, University of Agder, Apr2018, Kristiansand, Norway, 2018 a. 
STENDER, P. The use of heuristic strategies in modelling activities. ZDM: The International Journal on Mathematics Education, v. 50, n.1-2, p. 315-326, abril 2018.

SCHOENFELD, A. H. Mathematical Problem Solving. Orlando: Academic Press. 1985.

\section{Biografia Resumida}

Lourdes Maria Werle de Almeida: É professora da Universidade Estadual de Londrina desde 1985. Atua no curso de graduação em Matemática e é docente permanente do Programa de PósGraduação em Ensino de Ciências e Educação Matemática, tendo orientado inúmeras dissertações de mestrado e teses de doutorado. É bolsista de produtividade do CNPq e tem experiência na área de Matemática com ênfase em Educação Matemática. Tendo como foco de pesquisa a Modelagem Matemática na Educação Matemática, é autora ou co-autora em 60 artigos publicados em periódicos nacionais e internacionais da área. É autora ou co-autora de 04 livros sobre esta mesma temática e tem capítulos em livros nacionais e internacionais. É líder do GRUPEMAT- Grupo de Pesquisas sobre Modelagem e Educação Matemática cadastrado no CNPq e participa do Grupo de Pesquisa em Educação Matemática da UEL.

Link currículo lattes: http://lattes.cnpq.br/2660354136462141

Contato: lourdes@uel.br 